\title{
Fasciite craniana em paciente jovem
}

\author{
Francisco José de Alencar', Gerson Luis Medina Prado², Lina Gomes dos Santos ${ }^{3}$, \\ Maria Tereza Paraguassú Martins Guerra ${ }^{4}$, Maximiliano Ramos Pinto Carneiro 4 , \\ Camila Brito Falcão Pinheiro ${ }^{4}$, Márcia Beatriz de Jesus Lima ${ }^{4}$ \\ Universidade Estadual do Piauí (UESPI), Hospital São Marcos, Teresina, PI. \\ RESUMO \\ Fasciite craniana é uma lesão benigna rara do crânio, semelhante à fasciite nodular que ocorre mais \\ frequentemente na infância. Embora seja rara, essa lesão pode mimetizar entidades mais agressivas. \\ Clinicamente, apresenta-se como uma massa indolor e que raramente produz sintomas neurológicos. \\ Objetiva-se, aqui, relatar um caso de fasciite craniana em adolescente de 14 anos, descrevendo os \\ seus achados clínicos, de neuroimagem e anatomopatológicos, além de uma breve revisão da literatura.

\section{PALAVRA-CHAVE} \\ Fasciite craniana.

\section{ABSTRACT} \\ Cranial fasciitis in a young patient: case report and review of literature \\ Cranial fasciitis is a rare benign intracranial lesion, similar to nodular fasciitis, most frequently occurring \\ in children. Although rare, this lesion can mimic more aggressive entities. The clinical presentation \\ includes a solid painless lesion, rarely causing neurological symptoms. The purpose of this report is to \\ report a case of cranial fasciitis in a 14 year-old patient, describing relevant clinical, neuroimaging and \\ pathological findings as well as a brief literature review.

\section{KEY WORD} \\ Cranial fasciitis.
}

\section{Introdução}

Fasciite craniana (FC) é uma lesão benigna rara do crânio que ocorre quase exclusivamente na infância. Essa lesão é histologicamente idêntica à fasciite nodular. Embora seja rara, essa lesão pode mimetizar clínica e radiologicamente entidades mais agressivas. Clinicamente, apresenta-se como uma massa única, indolor e que raramente produz sintomas neurológicos. ${ }^{4}$

Objetiva-se, aqui, relatar um caso de FC em adolescente de 14 anos, descrevendo os seus achados clínicos, imagiológicos e anatomopatológicos, além de uma breve revisão da literatura.

\section{Relato do caso}

VRS, 14 anos de idade, sexo feminino, estudante, queixando-se de cefaleia de início há quatro anos, evoluindo com perda progressiva da acuidade visual direita. Encaminhada a serviço de referência, foi atendida por um neurocirurgião que, entre outros exames complementares, solicitou tomografia computadorizada (TC), a qual revelou a presença de uma lesão expansiva de comportamento agressivo com extensão craniofacial, de densidade intermediária e captando o meio de contraste (Figura 1).

A ressonância magnética (RM) demonstrou a natureza sólida da lesão com sinal isointenso/T1, hiperintenso/

1. Neurocirurgião.

2. Radiologista doutor em Medicina pela Universidade de Hirosaki, Japão (revalidado pela Universidade Federal do Rio de Janeiro - UFRJ), membro titular do Colégio Brasileiro de Radiologia e Diagnóstico por Imagem.

3. Patologista doutoranda em Biotecnologia pela Universidade Federal do Piauí (UFPI)/Renorbio, mestre em Ciências e Saúde pela UFPI, professora-assistente da Disciplina de Patologia Geral da Universidade Estadual do Piauí (UESPI).

4. Acadêmico de Medicina da UESPI. 
T2/FLAIR, captando o meio de contraste paramagnético, além de extenso componente meníngeo (Figura 2).

A paciente foi submetida à microcirurgia diagnóstica com suspeita de meningioma, tendo sido realizada craniectomia e retirada de fragmentos da lesão acometendo a meninge.

$\mathrm{O}$ estudo histopatológico demonstrou tecido fibroconjuntivo permeado por linfócitos, plasmócitos, histiócitos e neutrófilos, caracterizando infiltrado celular policlonal, confirmado por imunoistoquímica. As células foram positivas para CD20, CD30 e CD68; as células fusiformes foram positivas para actina de músculo liso, caracterizando, portanto, processo inflamatório consistente com FC.
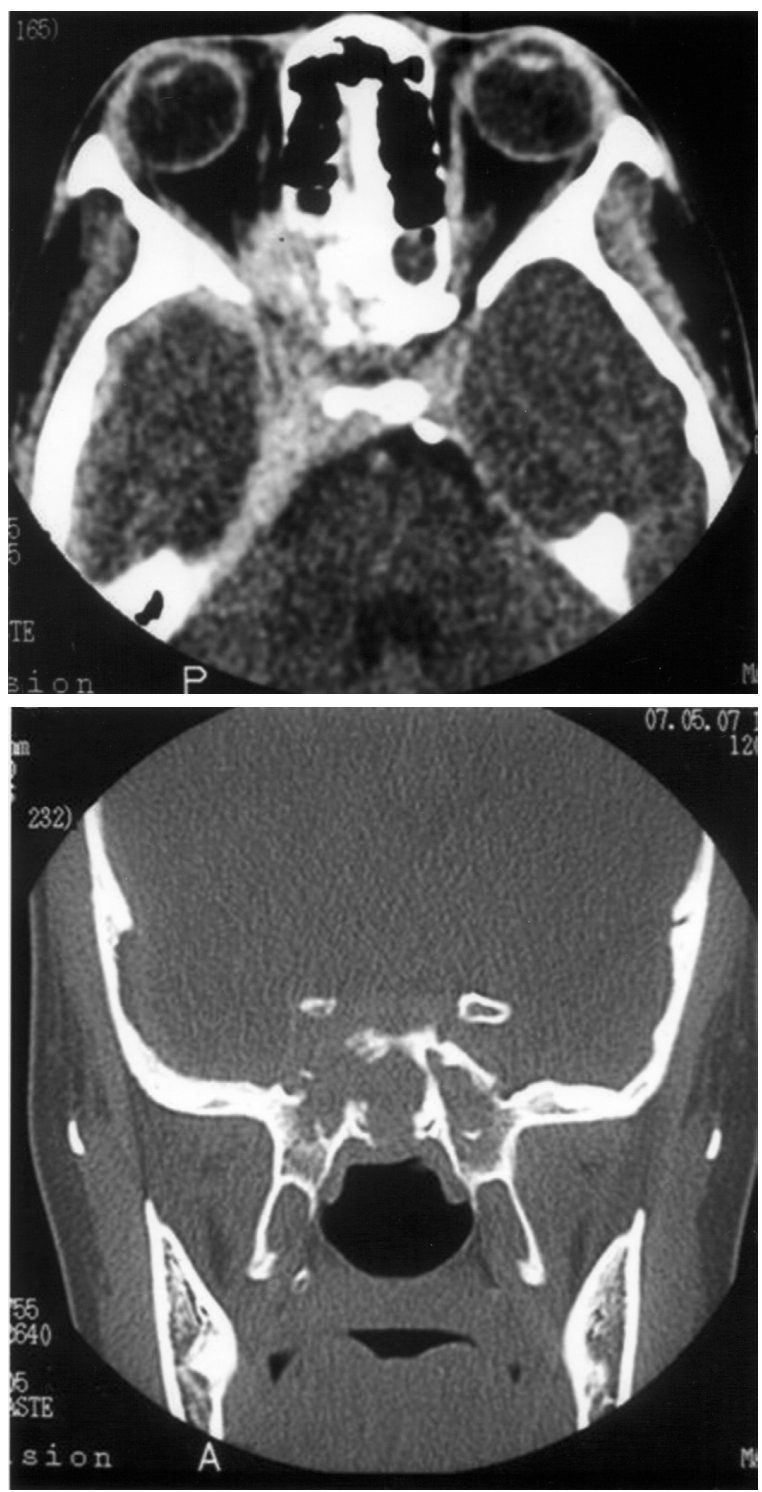

Figura 1-TC no plano axial demonstrando a presença de lesão expansiva captando contraste, espessamento dural ao longo da fossa temporal direita e plano esfenoidal, avançando em direção à órbita e etmoide, com sinais de erosão óssea; no plano coronal e em janela óssea evidenciam-se as áreas de erosão esfenoidal.
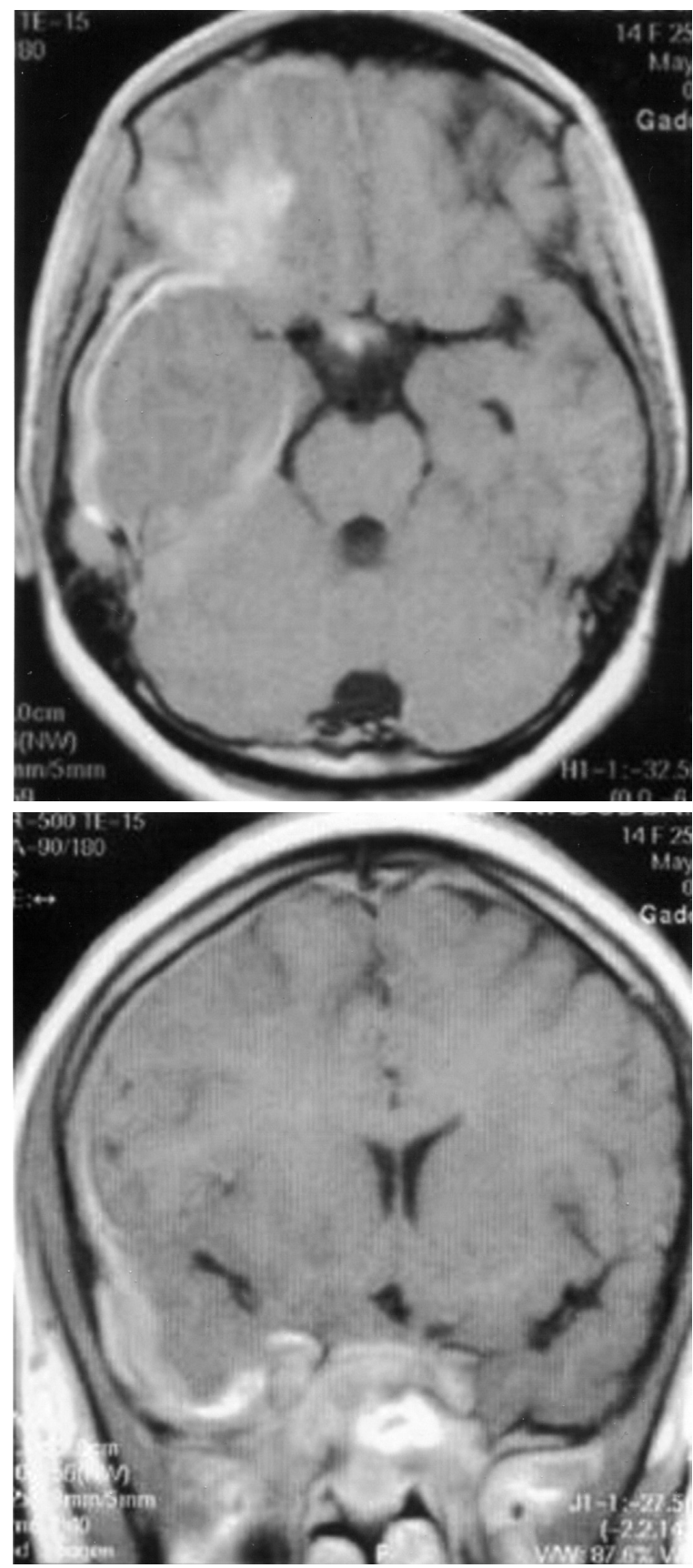

Figura 2-RM em cortes axial e coronal, ponderada em T1, após administração de gadolíneo, demonstra as áreas de realce anômalo ao longo da superficie dural direita, bem como componente parenquimatoso no lobo frontal.

Após o procedimento cirúrgico, a paciente não apresentou qualquer déficit, evoluindo neurologicamente assintomática. A TC de controle evidenciou apenas alterações morfológicas cranioencefálicas, ocasionadas pela abordagem da lesão. A conduta adotada, a partir de então, foi acompanhamento ambulatorial com exames de imagem para seguimento. 


\section{Discussão}

FC é uma lesão benigna rara, histologicamente idêntica à fasciite nodular, e corresponde a uma proliferação fibroblástica autolimitada das fáscias superficial e profunda. ${ }^{12}$ Essa lesão foi descrita pela primeira vez como parte do espectro da fasciite nodular em um estudo de 1980 feito por Lauer e Enzinger, ${ }^{6}$ em nove casos. Entre os achados que distinguem FC de FN estão a idade média de incidência da lesão e a sua topografia.

A FC é uma lesão extremamente rara, com apenas 40 casos descritos, até o ano de 2003, na literatura. ${ }^{4}$ A maioria desses casos aponta maior incidência dessa lesão em pacientes pediátricos. Segundo Marciano e cols. ${ }^{7}$ ocorre quase exclusivamente na infância, abaixo dos 6 anos, mas têm sido descritos casos de FC em adultos. Nenhum fator predisponente foi identificado; entretanto, muitos artigos citam o traumatismo prévio na área afetada como possível fator de desenvolvimento dessa entidade. ${ }^{1,6,8,10,11}$

Usualmente, a FC se manifesta como uma massa de crescimento rápido, consistência firme e não compressível. Quando presentes outros sintomas, estes são secundários ao efeito de massa e incluem diplopia, proptose, paralisia do nervo facial e hemiparesia. ${ }^{2,3,5,8,9}$

A excisão cirúrgica constitui o tratamento definitivo para essas lesões.

Os achados imagiológicos comumente revelam uma lesão única, lítica do crânio, que é mais bem definida com um componente de partes moles captante do meio de contraste, visto tanto em imagens de TC quanto de RM. O defeito lítico da calota craniana pode vir acompanhado de um halo esclerótico, embora neoformação óssea periosteal de aspecto agressivo também possa ser vista, além de calcificações no componente de partes moles. $\mathrm{O}$ achado mais comum inclui erosão da tábua óssea externa, embora extensão para a tábua interna com envolvimento dural tenha sido identificado em cerca de um terço dos casos relatados. ${ }^{4}$

O componente de partes moles visto na TC ou RM é levemente heterogêneo e demonstra intenso realce. Nas imagens de TC, as lesões também podem aparecer discretamente hiperatenuantes em relação ao tecido cerebral cortical. Na RM, as lesões apresentam-se isointensas em relação ao tecido cortical em imagens ponderadas em T1 e em T2. ${ }^{4}$

\section{Conclusão}

Esse caso exibe os aspectos clínicos, os de neuroimagem e morfológicos típicos dessa rara lesão, que deverá ser lembrada no diagnóstico diferencial de lesões cranianas.

\section{Referências}

1. Adler R, Wong CA. Cranial fasciitis simulating histiocytosis. J Pediatr. 1986;109:85-8.

2. Clapp CG, Dodson EE, Pickett BP, Lambert PR. Cranial fasciitis presenting as an external auditory mass. Arch Otolaryngol Head Neck Surg. 1997;123:223-5.

3. Iqbal K, Saqulain G, Udaipurwala IH, Ashraf J, Aijaz F, Jalisi M. Cranial fasciitis: presentation as a post-auricular mass. J Laryngol Otol. 1995;109:255-7.

4. Keyserling HF, Castillo M, Smith JK. Cranial fasciitis of childhood. AJNR. 2003;24:1465-7.

5. Kumon Y, Sakaki S, Sakoh M, Nakano K, Fukui K, Kurihara K. Cranial fasciitis of childhood: a case report. Surg Neurol. 1992;38:68-72.

6. Lauer DH, Enzinger FM. Cranial fasciitis of childhood. Cancer. 1980;45:401-6.

7. Marciano S, Vanel D, Mathieu MC. Cranial fasciitis in an adult: CT and MR imaging findings. Eur Radiol. 1999;9:1650-2.

8. Pagenstecher A, Emmerich B, van Velthoven $V$, Korinthenberg R, Volk B. Exclusively intracranial cranial fasciitis in a child: case report. J Neurosurg. 1995;83:744-7.

9. Pollack IF, Hamilton RL, Fitz C, Kassam A, Snyderman CH. Congenital reactive myofibroblastic tumor of the petrous bone: case report. Neurosurgery. 2001;48:430-5.

10. Ringsted J, Ladefoged C, Bjerre P. Cranial fasciitis of childhood. Acta Neuropathol. 1985;66:337-9.

11. Sato $Y$, Kitamura T, Suganuma Y, Kotani T, Hata J. Cranial fasciitis of childhood: a case report. Eur J Pediatr Surg. 1993;3:107-9.

12. Summers LE, Florez L, Berberian JM, Bhattacharjee M, Walsh J. Postoperative cranial fasciitis: report of two cases and review of the literature. J Neurosurg. 2007;106:1080-5.

Endereço para correspondência

Maria Tereza Paraguassú Martins Guerra

Rua 24 de Janeiro, 2139

64018-650 - Teresina, PI 